\title{
Jets of energetic particles generated by magnetic reconnection at a three-dimensional magnetic null
}

\author{
Silvia Dalla and Philippa K. Browning \\ School of Physics and Astronomy, University of Manchester, \\ PO Box 88, Manchester, M60 1QD, U.K. \\ email: s.dalla@manchester.ac.uk
}

\begin{abstract}
Magnetic reconnection is a candidate mechanism for particle acceleration in a variety of astrophysical contexts. It is now widely accepted that reconnection plays a key role in solar flares, and reconstructions of coronal magnetic fields indicate that three-dimensional (3D) magnetic null points can be present during flares. We investigate particle acceleration during spine reconnection at a $3 \mathrm{D}$ magnetic null point, using a test particle numerical code. We observe efficient particle acceleration and find that two energetic populations are produced: a trapped population of particles that remain in the vicinity of the null, and an escaping population, which leave the configuration in two symmetric jets along field lines near the spine. While the parameters used in our simulation aim to represent solar coronal plasma conditions of relevance for acceleration in flares, the fact that the 3D spine reconnection configuration naturally results in energetic particle jets may be of importance in other astrophysical situations. We also compare the results obtained for the spine reconnection regime with those for the other possible mode of $3 \mathrm{D}$ reconnection, fan reconnection. We find that in the latter case energetic particle jets are not produced, though acceleration is observed.
\end{abstract}

Keywords. acceleration of particles, Sun: flares

\section{Methodology and results}

We developed a numerical code that integrates the trajectories of a population of test particles in the magnetic and electric fields characteristic of 3D spine reconnection (Priest \& Titov 1996). Particles were injected at random locations on a sphere centered in the magnetic null, with initial energy distribution given by a Maxwellian. The parameters used in the simulation, aiming to represent flare-like conditions in the solar corona, are described in Dalla \& Browning (2006). Single particle trajectories in the configuration were previously studied by Dalla \& Browning (2005).

We find that spine reconnection at a $3 \mathrm{D}$ null can efficiently accelerate particles, and produces two energetic populations: a trapped one and an escaping one. Escaping particles leave the magnetic configuration along the spine line, in two symmetric jets. The average energy of the escaping population is lower than that of the trapped one. The spectrum produced can be approximated by a power law of spectral index 0.92 . The acceleration time scales of $\sim 60 \mathrm{~ms}$ obtained from our simulation, are broadly consistent with solar flare observations.

\section{References}

Dalla, S., \& Browning, P. K. 2005, A\& A, 436, 1103

Dalla, S., \& Browning, P. K. 2006, ApJ (Letters), 640, L99

Priest, E. R., \& Titov, V. S. 1996, Phil. Trans. R. Soc. Lond. A, 354, 2951 